\title{
Key Decision Just After 3 Months Following TKI Initiation in CML: Better and not Difficult
}

\author{
Ibrahim C. HAZNEDAROGLU
}

Hacettepe University Faculty of Medicine, Department of Hematology, Ankara, TURKEY

To the Editor,

The selection of the any first line TKI (tyrosine kinase inhibitor) and earlier switch at 3 months to a most powerful TKI based on the insufficient molecular response seem to be the most rational management strategy in chronic myeloid leukemia (CML). ${ }^{1,2}$ The depth of the molecular response and the time to achieve that response are important for the long-term prognosis of the CML patients. This is a key step for the prevention of disease progression in CML. ${ }^{3}$

The 3 months milestone molecular cut off value for BCR-ABL is $<10 \%$ IS. This data comes from several studies presented during the last year in the major scientific Hematology meetings. Four studies during the last ASH 2011 meeting [Marin et al., ASH abst.\# 785 (SPIRIT 2): The Predictive Value of Early Molecular Response in Chronic Phase CML Patients Treated with Dasatinib First Line Therapy., Hochhaus et al., ASH abst.\# 2767 (DASISION): Patients with Newly Diagnosed Chronic Myeloid Leukemia in Chronic Phase (CML-CP): Analysis of Molecular Response Kinetics in the DASISION Trial., Hanfstein et al., ASH abst.\# 783 (CML IV): Molecular and Cytogenetic Response After 3 Months of Imatinib Treatment Is Predictive for the Risk of Disease Progression and Death in Newly Diagnosed Chronic Myeloid Leukemia Patients - a Follow-up Analysis of the German CML Study IV., Nicolini et al., ASH abst.\# 1684: The Month Three Major Molecular Response in Chro- nic Phase Chronic Myeloid Leukemia on Imatinib 400, Nilotinib and Dasatinib Is a Major Prognostic Factor for Failure-Free and Progression-Free Survival.), Marin study in ASCO 2012 (Marin et al., JCO 2012. Assessment of BCR-ABL1 transcript levels at 3 months is the only requirement for predicting outcome for patients with chronic myeloid leukemia treated with tyrosine kinase inhibitors), Hochhaus study in EHA 2012 (Hochhaus et al., EHA 2012. Early BCR-ABL Transcript Levels Predict Future Molecular Response and Long-term Outcomes in Newly-diagnosed Patients With CML-CP: Analysis Of ENESTnd 3-year Data.) clearly indicated the importance of molecular response for predicting long-term prognosis at 3 months following the initiation of TKI in CML.

Second-generation TKIs induce earlier and deeper responses than imatinib and prevent more progressions as brilliantly outlined by Cortes. ${ }^{3}$ After a 12 month- follow-up, the rate of confirmed CCyR was higher with dasatinib than with imatinib $(77 \%$ vs. $66 \%, p=0.007)$, as well as the rate of CCyR observed on at least one assessment $(83 \%$ vs. $72 \%, \mathrm{p}=$ 0.001).

The rate of MMR was higher with dasatinib than with imatinib (46\% vs. $28 \%, \mathrm{p}<0.0001)$. Responses were achieved in a shorter time with dasatinib $(\mathrm{p}<$ $0.0001) .5$ patients receiving dasatinib $(1.9 \%)$ and 9 patients receiving imatinib $(3.5 \%)$ progressed to the accelerated or blastic crisis of CML. 
The two drugs showed similar safety profiles. When compared to imatinib, dasatinib induced significantly higher and faster rates of CCyR and MMR. As achievement of CCyR within 12 months is associated with better long-term, progressionfree survival, dasatinib may improve the long-term outcomes among patients with newly diagnosed CP-CML. ${ }^{4}$ Cumulative response rates by 24 months were: CCyR $86 \%$ and $82 \%$ for dasatinib and imatinib, respectievely, MMR $64 \%$ and $46 \%$, for dasatinib and imatinib repectively and BCR-ABL decreased to $0.0032 \%$ (4.5-log reduction) $17 \%$ and $8 \%$, for dasatinib and imatinib, respectively. Transformation to accelerated/blast phase CML occurred in $2.3 \%$ of patients with dasatinib vs $5.0 \%$ with imatinib. Based on the competing risk analysis, median time to CCyR was 3.2 months with dasatinib and 6.0 months with imatinib. The cumulative MMR rate by 24 months was $64 \%$ for dasatinib vs $46 \%$ for imatinib, and median time to MMR was 15 vs 36 months. 5 Patients with $>10 \%$ BCR-ABL levels at Month-3, had higher probability of giving/obtaining CCyR, MMR, MR4. ${ }^{5}$ within 3 years and lower probability of transformation to AP/BP at any time and higher probability of 3-year PFS and OS. ${ }^{6}$ Therefore, the proper switch to a most powerful TKI based on the insufficient monitorization responses shall be considered in the management of CML.

Molecular peripheral blood monitoring of CML is more sensitive and allows an early detection of potential relapse or patient non-compliance. Early deep responses predict for PFS and OS by any TKI determined by molecular monitoring. Selecting the earliest 3 months milestone molecular cut off value of BCR-ABL $<10 \% \mathrm{IS}^{1,2}$ is rational whether the first treatment had been started with the weakest TKI, generic Gleevec, or the strongest third generation TKI, investigational ponatinib. Earlier intervention at 3 months may be also useful for the early detection of multi-TKI (Imatinib, nilotinib, dasatinib, bosutinib) and transplant-resistant very aggressive CML types7 during the long-term clinicopathological course of CML?

Based on those perspectives, better long-term responses and outcomes may be foreseen by molecular responses obtained with the TKI, at the first 3month of the treatment. Further studies are defini- tely needed to reach an accurate communication on the prognostic role and the cut-off of BCR-ABL1 transcript level at 3 months in the 2 nd/3rd line therapy setting for CML patients with imatinib failure. The depth of the durable responses under any TKI and the time to achieve that response should be considered as the critical parameters for the better management of CML.3

\section{REFERENCES}

1. Hanfstein B, Muller MC, Hehlmann R, Erben P, Lauseker M, Fabarius A et al. Early molecular and cytogenetic response is predictive for long-term progressionfree and overall survival in chronic myeloid leukemia (CML). Leukemia 26: 2096-2102, 2012.

2. Marin D, Ibrahim AR, Lucas C, Gerrard G, Wang L, Szydlo RM et al. Assessment of BCR-ABL1 transcript levels at 3 months is the only requirement for predicting outcome for patients with chronic myeloid leukemia treated with tyrosine kinase inhibitors. J Clin Oncol 30: 232-238, 2012.

3. Cortes J. CML: the good, the better, and the difficult choices. Blood 120: 3866-3867, 2012.

4. Kantarjian $\mathrm{H}$, Shah NP, Hochhaus A. et al. Dasatinib versus Imatinib in Newly Diagnosed Chronic-Phase Chronic Myeloid Leukemia. N Engl J Med 362: 22602270, 2010.

5. Kantarjian $\mathrm{H}$, Shah NP, Cortes JE, et al. Dasatinib or imatinib in newly diagnosed chronic-phase chronic myeloid leukemia: 2-year follow-up from a randomized phase 3 trial (DASISION). Blood 119: 1123-1129, 2012.

6. Hochhaus A, Boqué C, Garelik BB, et al. Molecular Response Kinetics and BCR-ABL Reductions in Patients With Newly Diagnosed Chronic Myeloid Leukemia in Chronic Phase (CML-CP) Receiving Dasatinib Versus Imatinib: DASISION 3-Year Followup. 17th EHA Annual Congress; June 14-17, 2012. Abstract No. 1537.

7. Uz B, Bektas O, Eliacik E, et al. Allografting for Bosutinib, Imatinib, Nilotinib, Dasatinib, and Interferon Resistant Chronic Myeloid Leukemia without ABL Kinase Mutation. Case Reports Hematol 263725: doi: 10.1155/2011/263725, 2011

\section{Correspondence}

Prof. Dr. İbrahim C. HAZNEDAROGLU

Hacettepe Üniversitesi Tıp Fakültesi

Hematoloji Bilim Dalı

Sinhiye, ANKARA / TURKEY

Tel: (+90.312) 3051543

e-mail: haznedar@yahoo.com 